\title{
Genotype and phenotype in patients with Noonan syndrome and a RIT1 mutation
}

\author{
Karim Kouz ${ }^{1}$, Christina Lissewski², Stephanie Spranger, MD³, Diana Mitter, MD, \\ Angelika Riess, $\mathrm{MD}^{5}$, Vanesa Lopez-Gonzalez, $\mathrm{MD}^{6,7}$, Sabine Lüttgen, MD', \\ Hatip Aydin, $\mathrm{MD}^{8}$, Florian von Deimling, $\mathrm{MD}^{9}$, Christina Evers, $\mathrm{MD}^{10}$, \\ Andreas Hahn, MD ${ }^{11}$, Maja Hempel, MD ${ }^{1}$, Ulrike Issa, MD ${ }^{12}$, \\ Anne-Karin Kahlert, MD ${ }^{13,14}$, Adrian Lieb, MD ${ }^{15}$, \\ Pablo Villavicencio-Lorini, MD ${ }^{16}$, Maria Juliana Ballesta-Martinez, MD ${ }^{6,7}$, \\ Sheela Nampoothiri, MD ${ }^{17}$, Angela Ovens-Raeder, MD ${ }^{18}$, \\ Alena Puchmajerová, MD ${ }^{19}$, Robin Satanovskij, MD ${ }^{20}$, Heide Seidel, MD21, \\ Stephan Unkelbach, MD², Bernhard Zabel, MD²,23, Kerstin Kutsche, PhD ${ }^{1}$ and Martin Zenker, MD²
}

\begin{abstract}
Purpose: Noonan syndrome (NS) is an autosomal-dominant disorder characterized by craniofacial dysmorphism, growth retardation, cardiac abnormalities, and learning difficulties. It belongs to the RASopathies, which are caused by germ-line mutations in genes encoding components of the RAS mitogen-activated protein kinase (MAPK) pathway. RIT1 was recently reported as a disease gene for NS, but the number of published cases is still limited.

Methods: We sequenced RIT1 in 310 mutation-negative individuals with a suspected RASopathy and prospectively in individuals who underwent genetic testing for NS. Using a standardized form, we recorded clinical features of all RIT1 mutation-positive patients. Clinical and genotype data from 36 individuals with RIT1 mutation reported previously were reviewed.
\end{abstract}

Results: Eleven different RIT1 missense mutations, three of which were novel, were identified in 33 subjects from 28 families; codons 57, 82, and 95 represent mutation hotspots. In relation to NS of other genetic etiologies, prenatal abnormalities, cardiovascular disease, and lymphatic abnormalities were common in individuals with RIT1 mutation, whereas short stature, intellectual problems, pectus anomalies, and ectodermal findings were less frequent.

Conclusion: RIT1 is one of the major genes for NS. The RIT1-associated phenotype differs gradually from other NS subtypes, with a high prevalence of cardiovascular manifestations, especially hypertrophic cardiomyopathy, and lymphatic problems.

Genet Med advance online publication 21 April 2016

Key Words: hypertrophic cardiomyopathy; Noonan syndrome; oncogenic mutations; RASopathies; RAS-MAPK signaling pathway

\section{INTRODUCTION}

Noonan syndrome (NS; MIM 163950) is a relatively common autosomal-dominant disorder characterized by distinct craniofacial dysmorphism, postnatal growth retardation, and congenital cardiac defects such as pulmonary valve stenosis, atrial septal defects, and hypertrophic cardiomyopathy. Craniofacial features include broad forehead, hypertelorism, downslanting palpebral fissures, ptosis, and low-set posteriorly rotated ears. Affected individuals can also have pectus deformities, mild developmental delay, and/or learning difficulties and bleeding disorders; they are at risk of developing cancer, for example, juvenile myelomonocytic leukemia. ${ }^{1,2}$

\footnotetext{
The first two authors contributed equally to this work. The last two authors contributed equally to this work.

${ }^{1}$ Institute of Human Genetics, University Medical Center Hamburg-Eppendorf, Hamburg, Germany; ${ }^{2}$ Institute of Human Genetics, University Hospital Magdeburg, Magdeburg, Germany; ${ }^{3}$ Praxis für Humangenetik, Bremen, Germany; ${ }^{4}$ Institute of Human Genetics, University Hospital Leipzig, Leipzig, Germany; ${ }^{5}$ Institute of Medical Genetics and Applied Genomics, University of Tuebingen, Tuebingen, Germany; ${ }^{6}$ Sección de Genética Médica, Servicio de Pediatría, Hospital Clínico Universitario Virgen de la Arrixaca, IMIB-Arrixaca, Murcia, Spain; ${ }^{7}$ Centro de Investigación Biomédica en Red de Enfermedades Raras (CIBERER), Instituto de Salud Carlos III (ISCIII), Madrid, Spain; ${ }^{8}$ Department of Medical Genetics, Medical Faculty, Namık Kemal University, Tekirdag, Turkey; ${ }^{9}$ Sozialpädiatrisches Zentrum Coburg, Coburg, Germany; ${ }^{10}$ Institute of Human Genetics, Heidelberg University, Heidelberg, Germany; ${ }^{11}$ Department of Child Neurology, Justus-Liebig-University, Giessen, Germany; ${ }^{12}$ Facharztzentrum Pädiatrie und Humangenetik, Martin Luther Universität Halle-Wittenberg, Halle (Saale), Germany; ${ }^{13}$ Institut für Klinische Genetik, TU Dresden, Dresden, Germany; ${ }^{14}$ Department for Congenital Heart Disease and Pediatric Cardiology, University Hospital of Schleswig-Holstein, Kiel, Germany; ${ }^{15}$ Darmstädter Kinderkliniken, University Hospital Frankfurt, Frankfurt, Germany; ${ }^{16}$ Institute of Human Genetics, Martin Luther University Halle-Wittenberg, Halle (Saale), Germany; ${ }^{17}$ Department of Pediatric Genetics, Amrita Institute of Medical Sciences \& Research Centre, Cochin, India; ${ }^{18}$ Praxis für Humangenetik, München, Germany; ${ }^{19}$ Department of Biology and Medical Genetics, Charles University, 2nd Faculty of Medicine and University Hospital Motol, Prague, Czech Republic; ${ }^{20}$ Institut für Humangenetik, Klinikum rechts der Isar, Technische Universität München, München, Germany; ${ }^{21}$ Institute of Human Genetics, Ludwig-Maximilian University, Munich, Germany; ${ }^{22}$ Praxis für Kinder- und Jugendmedizin, Volkach, Germany; ${ }^{23}$ Centre for Pediatric and Adolescent Medicine, University Hospital Freiburg, Freiburg, Germany. Correspondence: Martin Zenker (martin.zenker@med.ovgu.de) Or Kerstin Kutsche (kkutsche@uke.de) 
NS is a genetically heterogeneous disorder; to date, germ-line mutations in more than 10 genes have been discovered. The three major genes are PTPN11, mutated in 50\% of cases (MIM 176876), ${ }^{3}$ SOS1 (10-15\%; MIM 182530), ${ }^{4,5}$ and RAF1 (5-10\%; MIM 164760). ${ }^{6,7}$ RIT1 (MIM 609591) was more recently identified as another causative gene for NS. ${ }^{8}$ The remaining causative genes for NS are KRAS (MIM 190070), ${ }^{9}$ NRAS (MIM 164790), ${ }^{10}$ RRAS (MIM 165090), ${ }^{11}$ CBL (MIM 165360), ${ }^{12}$ SOS2 (MIM 601247), and LZTR1 (MIM 600574), ${ }^{13}$ which are rarely mutated in NS. Mutations of SHOC2 (MIM 602775) account for the NS-like disorder with loose anagen hair (MIM 607721). ${ }^{14}$ Two genes, RASA2 (MIM 601589) and A2ML1 (MIM 610627), have recently been reported with mutations in a few individuals with NS-like phenotypes, ${ }^{15,16}$ but this finding has not yet been replicated by others. Mutations in BRAF and MAP2K1 that are usually associated with cardio-facio-cutaneous syndrome (MIM 115150) have occasionally been reported in NS. ${ }^{17-19}$

All causative genes for NS, except LZTR1 and A2ML1, encode components or regulators of the well-studied RAS mitogen-activated protein kinase (MAPK) signaling pathway. This pathway is critically involved in cell proliferation, differentiation, survival, and senescence. ${ }^{20} R A S$ genes constitute a multigene superfamily that includes HRAS, KRAS, NRAS, RRAS, and RIT1. They code for monomeric G proteins, which cycle between a GTP-bound active state and a GDP-bound inactive state. ${ }^{21}$ The RAS-MAPK signal transduction cascade is essential for normal mammalian development, implying that dysregulation of this pathway has severe consequences in embryonic development. NS and other clinically overlapping diseases such as cardio-facio-cutaneous syndrome, Costello syndrome (MIM 218040), Legius syndrome (MIM 611431), and neurofibromatosis type 1 (MIM 162200) constitute the group of RASopathies, a class of developmental disorders caused by increased signal flux through the RAS-MAPK pathway.22

So far, 11 different missense mutations in the RIT1 gene have been reported in a total of 36 individuals with NS. ${ }^{8,15,23-27}$ Enhanced transactivation of the transcription factor ELK1, which is activated by the kinase ERK2 of the MAPK cascade, has been shown upon overexpression of NS-associated RIT1 mutant proteins in NIH 3T3 cells. ${ }^{8}$ Further evidence of a gain-of-function effect of the NS-related RIT1 mutations came from MEK-ERK activation assays. The amino acid substitutions p.Ala57Gly and p.Met90Ile caused increased phosphorylation of the MAP kinases MEK and ERK when the respective RIT1 mutant was expressed in PC6 cells. ${ }^{24}$ The phenotype displayed by RIT1 mutation carriers is fitting of NS, with no obvious genotype-phenotype correlations identified to date ${ }^{8,23-25}$ Here, we report 33 subjects with a RIT1 mutation and review clinical features of our and previously reported cases.

\section{MATERIALS AND METHODS}

\section{Subjects and phenotyping}

Study subjects were referred to two genetic centers (University Hospital of Magdeburg and University Medical Center Hamburg-Eppendorf) for molecular diagnosis of NS. The study cohort comprised 310 individuals who had previously tested negative for other NS-causative genes. It consisted of subjects exhibiting variable clinical features within the RASopathy phenotypic spectrum, including a considerable number of cases with an attenuated or atypical phenotype. Additionally, individuals with suspected NS who were newly referred to our center for diagnostic testing and turned out to have a RIT1 mutation were included in the genotype-phenotype analysis. Clinical data and samples for all individuals were obtained with informed consent of the patients' parents/legal guardians or the patients themselves, including written consent to use photographs in this report, according to the Declaration of Helsinki and the national legal regulations (e.g., the German Genetic Diagnosis Act (GenDG)).

All affected individuals were personally examined by a physician who is experienced in clinical syndromology, and photos were reviewed by human geneticists with specific clinical expertise in RASopathies (M.Z., K. Kutsche, and S.S.). Standardized phenotypic data were collected using the electronic questionnaire of the NSEuroNet database (http://www.nseuronet.com; more details are provided in the legend to Supplementary Table S4 online). Standard deviations (SD) of the height were calculated using the pediatric calculator ped(z) (https://www.pedz.de/en/ welcome.html). For the majority of patients who were of German descent, the standard German curves were used as reference. ${ }^{28}$ For individuals of non-German origin, the standards as published by the World Health Organization (birth to 2 years of age) and the Centers for Disease Control and Prevention (http://www. cdc.gov/growthcharts/) (2-18 years of age) were used as reference. The craniofacial phenotype was classified on a subjective basis as typical, suggestive, or atypical for Noonan syndrome. ${ }^{29}$

\section{Molecular analysis}

DNA was isolated from leukocytes by standard procedures. The coding region and exon-intron boundaries of the RIT1 gene (six exons) (GenBank:NM_006912.5; encodes isoform 2 with a shorter $\mathrm{N}$-terminus compared with isoform 1) was amplified from genomic DNA. Primer sequences are available on request. Amplicons were directly sequenced using the ABI BigDye Terminator Sequencing Kit (Applied Biosystems, Darmstadt, Germany) and an automated capillary sequencer (ABI 3500; Applied Biosystems). Sequence electropherograms were analyzed using the Sequence Pilot software SeqPatient (JSI medical systems, Ettenheim, Germany). Genotyping was performed with the AmpFLSTR SGM plus PCR Amplification Kit (Applied Biosystems) to confirm paternity and maternity. RIT1 variants were described according to both the long and short transcript variants (mRNA RefSeqs NM_001256821.1 and NM_006912.5) and isoforms (protein RefSeqs NP_001243750.1 and NP_008843.1) in Supplementary Table S1 online.

\section{RIT1 mutations}

\section{RESULTS}

We identified 11 different RIT1 missense mutations in 28 unrelated patients (Table 1). Pathogenicity of RIT1 variants was 


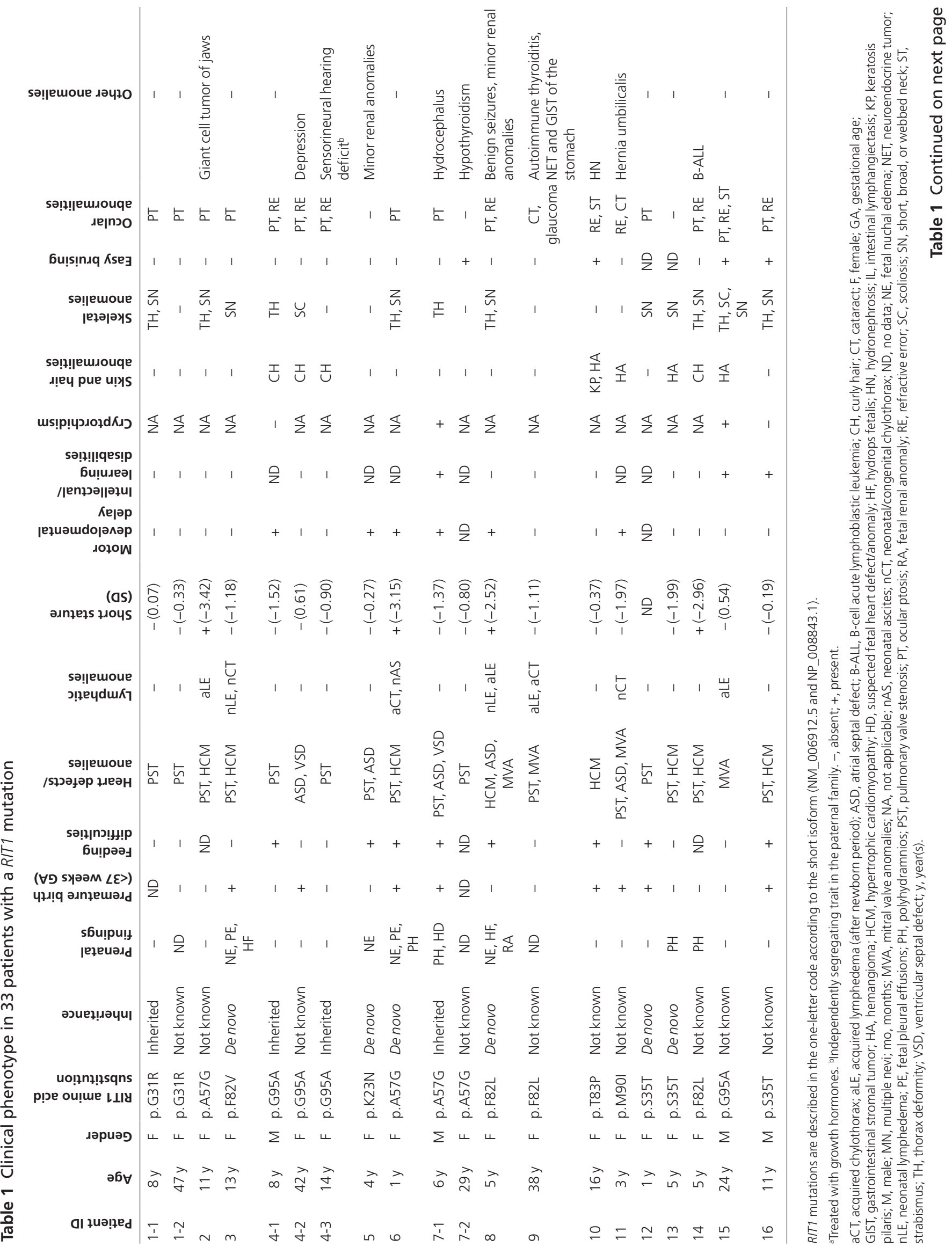




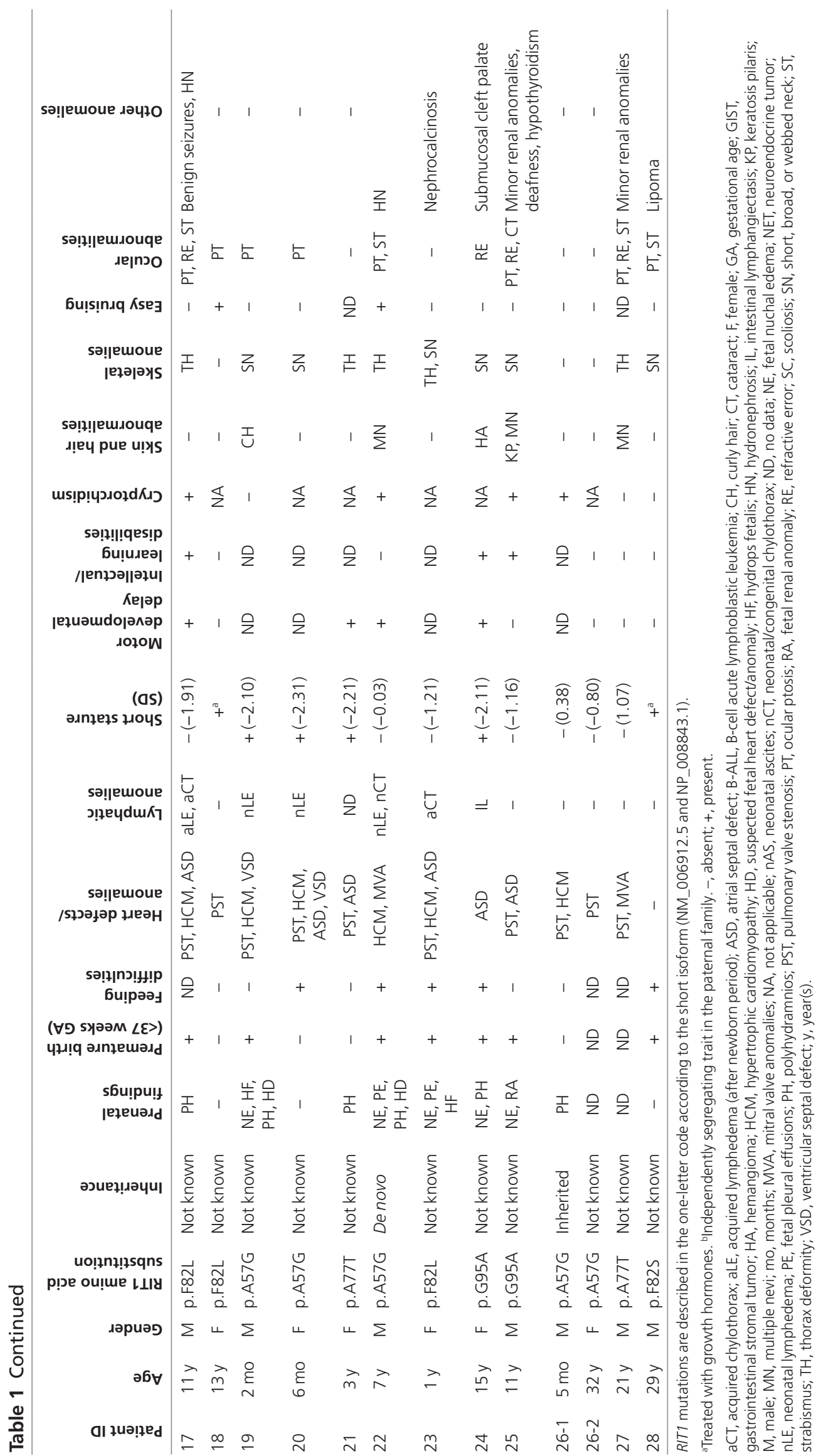




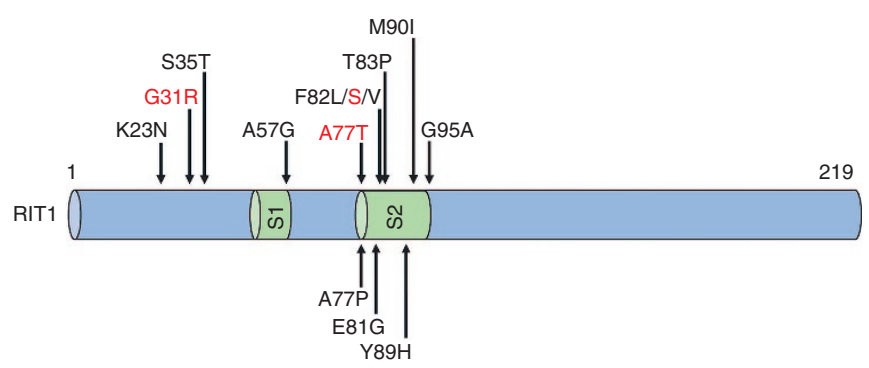

Figure 1 RIT1 domain structure and NS-associated amino acid substitutions. The first and the last amino acids of the small RIT1 isoform (according to protein Ref Seq NP_008843.1) are given above the domain structure. Switch I (S1) and switch II (S2) domains are indicated as green barrels. Amino acid substitutions identified in the two cohorts reported here are given in one-letter code above the structure and those reported previously and not found in this study are below the structure. Novel mutations are highlighted in red.

classified according to the guidelines of the American College of Medical Genetics and Genomics (Supplementary Table S1 online). ${ }^{30}$ Twenty-one index cases were identified in a retrospective cohort of 310 patients with NS who previously tested negative for other known NS genes, and 7 RIT1 mutation-positive patients were prospectively recruited from individuals who underwent molecular diagnostic testing for NS since September 2013. Three of the missense changes have not been described previously: c.91G $>$ A/p.(Gly31Arg), c.229G $>$ A/p.(Ala77Thr), and c.245T $>\mathrm{C} / \mathrm{p}$.(Phe82Ser); however, a different RIT1 mutation affecting codon 77 (c.229G $>\mathrm{C} / \mathrm{p}$.(Ala77Pro)) and two others changing codon 82 (c.244T $>$ G/p. . (Phe82Val) and c.246T $>$ G/ p.(Phe82Leu)) have already been reported (Figure 1). ${ }^{8,15}$ The p.(Gly31Arg) variant is absent from the databases dbSNP, 1,000 Genomes Project, and ExAC Browser databases and is predicted to be damaging by the Combined Annotation Dependent Depletion scoring system (data not shown). ${ }^{31}$ For seven mutations, de novo occurrence was demonstrated by parental testing. Four mutations were familial, with five additional affected family members being confirmed carriers of the respective mutation (patients 1-2, 4-2, 4-3, 7-2, and 26-2), including a mother and her daughter carrying the novel mutation p.(Gly31Arg) (Table 1 and Supplementary Table S1 online). These familial cases expand the number of RIT1 mutation-positive individuals to 33 . Segregation of the RIT1 mutation could not be investigated in 21 individuals because DNA samples for one or both parents were not available (Table 1 and Supplementary Table S1 online).

\section{Phenotype analysis}

Clinical findings for 33 individuals with a RIT1 mutation (22 females and 11 males) are summarized in Table 1. Overall, the clinical phenotype is characteristic of NS. In all subjects, the craniofacial phenotype was evaluated by experienced RASopathy specialists (M.Z., K.Kutsche, and S.S.) on the basis of personal examination or photographs. In all of the patients we found variable combinations of craniofacial dysmorphisms characteristic of NS, such as broad forehead, hypertelorism, downslanting palpebral fissures, ptosis, broad nasal bridge, low-set ears, and short neck; all faces were classified as typical or suggestive of NS (Figure 2 and data not shown). Prenatal abnormalities were recorded in 15/28 (54\%) patients.

The most common findings were polyhydramnios $(n=10)$ and fetal nuchal edema $(n=9)$. Six affected fetuses had either isolated pleural effusions or hydrops fetalis. A fetal heart defect/ anomaly was suspected in only three cases. Premature birth was common: 15/29 (52\%) were born before the week 37 of gestation, and five of them were born before week 34 of gestation. In 13 of 27 individuals (48\%), feeding difficulties in infancy were reported, and six patients required gavage feeding. In the latter case, infants were born prematurely, with one exception (data not shown). Two children had gastroesophageal reflux. Almost all patients had congenital heart defect (32/33; 97\%), with pulmonary valve stenosis being the most common anomaly $(26 / 33 ; 79 \%)$. A high rate of hypertrophic cardiomyopathy (HCM) was also observed (14/33; 42\%); 12/33 (36\%) had septal defects, mainly atrial septum defect. Twenty patients underwent either catheter intervention or open surgery for congenital heart defects (data not shown).

Postnatal lymphatic anomalies were noted in 13/32 (41\%), including several cases with neonatal lymphedema $(n=5)$ and congenital chylothorax $(n=3)$. Notably, acquired lymphedema (occurring after the newborn period), such as lymphedema of lower limbs and genitalia, acquired chylothorax, or a combination of both, was found in 6/32 individuals, and another (patient 24) had intestinal lymphangiectasis with protein-losing enteropathy, resulting in $22 \%$ with late-onset lymphatic complications that lead to significant morbidity. Short stature (height $\mathrm{SD}$ below -2.00 ; third percentile) was found in only $10 / 32$ individuals (31\%), and another 5 patients had height below -1.25 SD (10th percentile). Two patients had undergone growth hormone treatment. None of the eight adult RIT1 mutation carriers in our cohort had a body height below $-1.25 \mathrm{SD}$ (10th percentile). Motor developmental delay was observed in 10/27 subjects (37\%) and was frequently associated with muscular hypotonia. In 5 of them, delay in the achievement of motor milestones was mild (unaided sitting between 9 and 12 months and/or unaided walking between 18 and 24 months) or not specified. Six individuals showed moderate delay in motor milestones (unaided sitting between 12 and 18 months and/or unaided walking between 24 and 36 months), but 5 of them had a history of premature birth.

Learning or intellectual disabilities were reported in $6 / 22$ (27\%) of our RIT1 mutation-positive subjects aged 5 years or older. In four of six individuals, mental disabilities were mild (Supplementary Table S2 online). Learning difficulties were associated with attention-deficit disorder in three individuals (Supplementary Table S2 online). Six out of 11 male patients (55\%) had cryptorchidism. Skin and hair abnormalities were found in 13/33 individuals (39\%), with curly hair and cutaneous hemangioma as the most common findings in five individuals each. Keratosis pilaris was found in only 2 patients. A short, broad, and/or webbed neck was present in 16/33 (48\%) individuals and pectus deformities in 14/33 (42\%). In 6 out of 29 individuals (21\%), easy bruising was reported. 

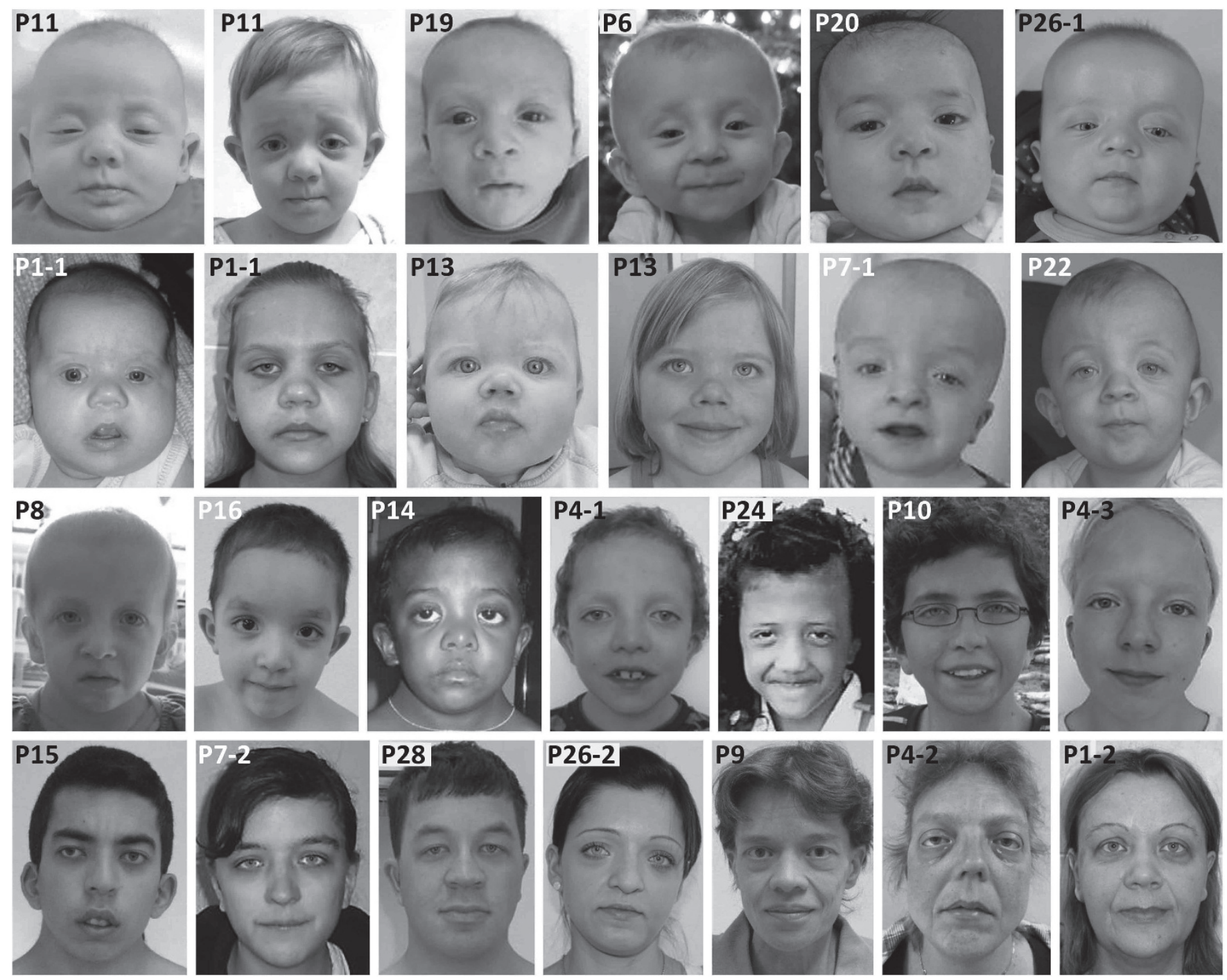

Figure 2 Craniofacial phenotype in patients with RIT1 mutation. Facial photographs of 23 individuals from our cohort displaying the spectrum of RIT1 mutation-associated facial dysmorphism from infancy to adulthood. Patients 11, 1-1, and 13 are shown at different ages (infancy vs. 2.6, 8.5, and 4.7 years, respectively) to document evolution of the craniofacial phenotype. All affected individuals exhibit recognizable dysmorphic features of Noonan syndrome, although the expression is quite variable. Patient IDs refer to Table 1.

Ocular abnormalities were seen quite often (26/33 individuals; $79 \%$ ), with ptosis as the most common feature observed in $22 / 33$ subjects (67\%). Ocular ptosis was significant in three individuals for whom surgical correction was either planned or performed. Refractive errors were present in 13/33 (39\%). Three individuals had cataracts. Four patients developed benign or malignant neoplasias, including multiple giant cell tumors of the jaws in patient 2, B-cell acute lymphoblastic leukemia in patient 14 , a gastrointestinal stromal tumor and a neuroendocrine tumor in patient 9 , and a lipoma in patient 28 . Three patients had hydronephrosis requiring surgery, and four others showed minor renal anomalies. Three individuals had documented hypothyroidism or autoimmune thyroiditis. Two subjects had deafness requiring hearing aids; however, in patient $4-3$, this is possibly a different genetic trait.

\section{DISCUSSION}

Herein we present a large contiguous cohort of patients with NS caused by RIT1 mutation. We identified 21 unrelated RIT1 mutation-positive patients among 310 individuals with a clinical diagnosis of NS or a RASopathy-like disorder who were negative for a mutation in the previously identified NS-linked genes, providing a mutation detection rate of $6.8 \%$ in this cohort. An additional seven RIT1 mutation-positive cases were identified prospectively among patients referred for molecular diagnostic testing. We identified RIT1 mutations in individuals with a clinical diagnosis of NS; none of them had been suspected of having cardio-facio-cutaneous or Costello syndrome. Two previous studies by Aoki et al. (2013) and Bertola et al. (2014) reported a RIT1 mutation detection rate of approximately $9 \%$ in cohorts of patients who had tested negative for mutations in the previously known genes. By contrast, Gos 
et al. (2014) found that only $3.8 \%$ of their mutation-negative cases with NS carried a RIT1 mutation. The differences in the detection rates, however, are likely to be explained by more or less strict clinical inclusion criteria, and these figures do not reflect the prevalence of RIT1 alterations in an unselected cohort of NS-affected subjects. To provide a better estimate of the contribution of RIT1 mutations to NS, we calculated the relative frequency of mutations in the NS-linked genes among 507 individuals who tested positive for NS at the University of Magdeburg since 2009: PTPN11, 54\%; SOS1, 18\%; RAF1, 11\%; RIT1, 5\%; KRAS, 3\%; CBL, 2\%; and NRAS, 1\% (M.Z., unpublished data). This cohort is not free from any bias, and, compared with an unselected patient population, these figures may be an overestimation for mutations in SOS1 and RAF1, but an underestimation for RIT1 alterations. Nevertheless, these numbers indicate that RIT1 mutations are quite common, and RIT1 is among the four major genes for NS, accounting for at least $5 \%$ of molecularly confirmed NS-affected cases.

In the 28 RIT1 mutation-positive index patients reported here, the majority of mutations were found to affect codon 82 $(n=8)$ (Table 1), giving rise to amino acid substitution of phenylalanine to valine, leucine, or serine (Figure 1). The two other most common RIT1 codons mutated in NS-affected individuals are $57(n=7)$ and $95(n=4)$ (Table 1$)$. Clustering of diseasecausative variants at codons 57,82 , and 95 has already been observed. ${ }^{8,23}$ By combining the published RIT1 germ-line alleles with those reported here, we calculated that $23 \%$ of the substitutions occurred at codon $57,22 \%$ occurred at codon 82 , and $22 \%$ occurred at codon 95 , yielding a total of $67 \%$ of all RIT1 mutations affecting one of these three triplets, whereas alterations of the eight other codons $(23,31,35,77,81,83,89$, and 90) together account for $33 \%$ of identified mutations (this study and refs. ${ }^{8,15,23-27}$ ). These data are in line with gain-of-function mutations in other genes found in NS-affected individuals because they cluster at codons encoding highly conserved amino acids in functionally important domains.

The RIT1 mutation p.(Gly31Arg) identified in this study is novel and alters a functionally relevant amino acid residue. Glycine 31 in RIT1 corresponds to glycine 13 in the RAS GTPases. Glycine 13 of HRAS is mutated in individuals with Costello syndrome. ${ }^{32}$ These findings, together with pathogenicity prediction and segregation of the variant with disease (Supplementary Table S1 online), provide strong evidence for causality of the RIT1 mutation p.(Gly31Arg).

To better define the phenotype associated with a RIT1 mutation, we summarized the clinical features observed in our 33 patients and reviewed those of 36 individuals described in the literature (Supplementary Table S3 online)., ${ }^{8,1523-27}$ We also compared the frequency of phenotypic features in our RIT1 mutation-positive cohort with patients harboring a PTPN11, SOS1, or RAF1 mutation, whose phenotype data had been collected using the same standardized form (NSEuroNet database; Supplementary Table S4 online). In more than half of the RIT1 mutation-positive cases, prenatal abnormalities were observed, including polyhydramnios as the most common finding and also fetal nuchal edema, fetal pleural effusions, and hydrops fetalis. The frequency of prenatal abnormalities was comparable between our cohort and previously published cases with RIT1 mutation (Supplementary Table S3 online). Compared with other NS genes, especially PTPN11, in which mutations account for the largest fraction of NS-affected cases, a significantly higher frequency of fetal nuchal edema (32\%), fetal chylothorax, and/or fetal hydrops (21\%) was observed (Supplementary Table S4 online). Notably, RIT1 mutation-positive individuals from our cohort also had a higher incidence $(22 \%)$ of lymphatic disorders occurring later in life, which was significant in comparison to patients with PTPN11 and RAF1 mutations (Supplementary Table S4 online). Three cases with RIT1 mutation and acquired lymphatic disorders were also reported in the literature (Supplementary Table S3 online).

A significant proportion of all patients with a RIT1 germline mutation in our cohort (48\%) and previously reported cases (59\%) had feeding difficulties (Supplementary Table S3 online); however, in our cohort we could relate the more severe feeding difficulties to premature birth (data not shown). Overall feeding issues appear to occur less frequently in individuals with a RIT1 mutation compared with NS-affected individuals linked to other genes; ${ }^{33}$ however, this difference does not reach statistical significance in our data set (Supplementary Table S4 online). Cardiovascular abnormalities were seen in almost all individuals with a RIT1 alteration (combined frequency 66/68; 97\%; Supplementary Table S3 online). This is significantly higher than in patients with PTPN11 mutation (79\%; Supplementary Table S4 online), and also higher than the frequency reported for NS overall (81\%; no patients with RIT1 mutation included). ${ }^{34}$ The most prevalent heart defect was pulmonary or pulmonary valve stenosis found in a total of 54 out of 68 RIT1 mutation-positive individuals (79\%) (Supplementary Table S3 online) compared with a prevalence of $56 \%$ in patients with PTPN11 mutation, $15 \%$ in those with RAF1 mutation (Supplementary Table S4 online), and 57\% in molecularly confirmed patients with NS overall. ${ }^{34}$ A high incidence of HCM in NS-affected subjects with RIT1 mutation has already been noticed by Aoki et al. ${ }^{8}$ There is a total prevalence of HCM of 52\% (33/63) in individuals with a RIT1 mutation (Supplementary Table S3 online) compared with $16 \%$ in reported individuals with a molecularly confirmed diagnosis of NS in general (without RIT1). ${ }^{34}$

Although HCM was somewhat less frequent in our patient cohort with RIT1 alteration (42\%) (Supplementary Table S4 online), we found significant differences compared with individuals with PTPN11 or SOS1 mutation (12\% each) in our data set (Supplementary Table S4 online), which is consistent with published data. ${ }^{33}$ These findings corroborate that RIT1 is the second most important NS gene associated with HCM; only RAF1 germ-line mutations show a higher association with HCM (approximately 80\%) (Supplementary Table S4 online). ${ }^{33}$ NS-affected individuals with HCM show significant early mortality ( $22 \%$ by the age of 1 year). ${ }^{35}$ However, there was no instance of cardiac death related to HCM in our cohort of RIT1 
mutation-positive patients or in other reported patients, , $^{8,15,23-27}$ suggesting that HCM associated with RIT1 mutations has a more benign course. Atrial or ventricular septal defects were found in a proportion of subjects with RIT1 mutation, which is comparable to individuals with mutations in other genes (Supplementary Table S4 online). ${ }^{33}$ Short stature, which is a common feature in NS, ${ }^{36}$ was consistently found at a relatively low frequency, affecting a total of only 24 of 64 individuals with RIT1 alteration (38\%) (Supplementary Table S3 online). The frequency of short stature in RIT1 mutation-positive individuals is significantly lower than in patients with an alteration in PTPN11, SOS1, and RAF1 in our data set (Supplementary Table S4 online). Accordingly, Kobayashi et al. ${ }^{33}$ recorded a higher incidence of short stature, particularly in patients with a RAF1 (82\%) or PTPN11 (56\%) mutation.

Consistent with our data (Supplementary Tables S2 and $\mathrm{S} 3$ online), a relatively low percentage of subjects with RIT1 mutation was recorded to have intellectual/learning disabilities (13/49; 27\% in total; Supplementary Table S3 online), which corresponds well to $21 \%$ of individuals with SOS1 mutation in our cohort (Supplementary Table S4 online) and 18\% reported in the literature. ${ }^{33}$ Conversely, in patients with $R A F 1$ and PTPN11 mutation, frequencies of $43 \%$ and $40 \%$, respectively, were recorded for intellectual/learning disabilities in our data (Supplementary Table S4 online), which is consistent with published data. ${ }^{33}$ However, differences in the overall prevalence of intellectual/learning disabilities between patients with RIT1, PTPN11, SOS1, and RAF1 mutation in our cohort did not reach statistical significance, probably because of a limited sample size (Supplementary Table S4 online). A more detailed analysis of the degree of intellectual impairment showed that the recorded deficits were usually mild in the RIT1 mutation-positive patients described here. Specific learning disabilities were uncommon, but numbers were too small to achieve statistically significant differences (Supplementary Table S2 online).

Ectodermal abnormalities such as curly hair and hyperkeratosis are relatively uncommon in RIT1 mutation-positive subjects from our cohort as well as in previously published cases (Supplementary Tables S3 and S4 online). Significantly higher frequencies of ectodermal abnormalities were recorded in individuals with SOS1 mutation in our data set (Supplementary Table S4 online), which fits well with data from the literature. ${ }^{33}$ Pectus deformities and a short, broad, or webbed neck are consistently observed in NS-affected individuals with mutations in different genes. ${ }^{24,33}$ We observed significantly lower frequencies of these anomalies in patients with RIT1 mutation compared with SOS1 and RAF1 (Supplementary Table S4 online).

Bleeding diathesis was rarely observed in patients with RIT1 mutation $(21 \%)$ in our cohort, but it is not significantly different from the rate of patients carrying mutations in PTPN11, SOS1, and $R A F 1(33,21$, and $17 \%$, respectively; Supplementary Table S4 online). In our cohort, bleeding problems were rather mild and without any severe complications (data not shown). Ocular ptosis was seen quite often in our RIT1 mutation-positive cohort (67\%) (Supplementary Table S4 online) and in a total of 36 out of 56 (64\%) individuals with RIT1 alteration (Supplementary Table S3 online). This association is quite similar in all other patient groups with molecularly confirmed NS (60-78\% in our cohort in Supplementary Table S4 online and $62-79 \%$ reported in ref. $\left.{ }^{33}\right)$. However, severe ptosis requiring surgical interventions appears to be rare in RIT1-mutated patients (three individuals in our cohort and no documentation in previous studies).

Somatic mutations in RIT1 have recently been identified in myeloid malignancies and lung adenocarcinomas. ${ }^{37-39}$ The RIT1 mutational spectrum found in human cancers significantly overlaps with RIT1 germ-line alleles: all oncogenic mutations clustered around glutamine 79 in the switch II region (p.(Ala77Pro), p.(Glu81Gly), p.(Phe82Leu/Val), and $\mathrm{p}$.(Met90Ile)) and induced cellular transformation. ${ }^{37-39}$ A recent study of cancer spectrum and frequency in patients with RASopathies demonstrated an 8.1-fold increased risk of all childhood tumors in NS-affected children, ${ }^{40}$ but because this study included only patients with a molecularly confirmed diagnosis of a RASopathy before 2013, no cases with a RIT1 mutation were included. We report the second case of RIT1 mutation-associated NS with acute lymphoblastic leukemia and multiple giant cell lesions of the jaws (Table 1 and refs. ${ }^{8,25}$ ), suggesting that individuals carrying a RIT1 germ-line mutation might be at increased risk for developing these types of neoplasia. Two other patients in our cohort developed malignant or benign tumors, thus bringing the total number of RIT1 mutation-positive individuals with any type of neoplasia to 6/68 (9\%) (Supplementary Table S3 online), including three cases of malignancy. The three subjects with a malignant tumor had alterations affecting codons 81 and 82 , which are common sites of somatic RIT1 mutations. ${ }^{37-39}$ The two patients reported with giant cell tumors of the jaws (our cohort and Bertola et al. (2014)) shared the RIT1 mutation p.(A57G). In summary, these findings indicate that Noonan syndrome-associated RIT1 germ-line mutations do confer susceptibility to neoplasia; however, further studies are needed to determine the risk for malignancies in this particular NS patient group.

\section{Conclusion}

We conclude that RIT1 is one of the four most frequently mutated genes in patients with a clinical phenotype of NS. Using careful clinical evaluation of our patient cohort and reviewing previously reported cases, we delineated in more detail the clinical characteristics of RIT1-associated NS. We found a high frequency of cardiovascular abnormalities with particular risk of HCM and a predisposition to lymphatic problems, whereas short stature, developmental delay, bleeding diathesis, and ectodermal abnormalities occurred at lower frequencies compared with NS in general. We add the three novel amino acid substitutions p.(Gly31Arg), p.(Ala77Thr), and p.(Phe82Ser) to 11 already reported NS-associated RIT1 alterations. The majority of RIT1 germ-line mutations cluster at codons 57, 82, and 95, and the mutation spectrum in NS-affected individuals significantly overlaps with that of somatic mutations in tumors. We 
found a possible hint of increased risk of neoplasia in subjects with RIT1 mutation-associated NS.

\section{SUPPLEMENTARY MATERIAL}

Supplementary material is linked to the online version of the paper at http://www.nature.com/gim

\section{ACKNOWLEDGMENTS}

We are grateful to the patients and their families who contributed to this study. We thank Inka Jantke and Dennis Zorndt for skillful technical assistance, Frederike L. Harms for help with RIT1 sequencing, and Uta Meyer zum Büschenfelde for fruitful discussions. This work was supported by grants from the Deutsche Forschungsgemeinschaft (DFG) (KU 1240/9-1 to K.Kutsche and ZE 524/10-1 to M.Z.).

\section{DISCLOSURE}

The authors declare no conflict of interest.

\section{REFERENCES}

1. Romano AA, Allanson JE, Dahlgren J, et al. Noonan syndrome: clinical features, diagnosis, and management guidelines. Pediatrics 2010;126:746-759.

2. van der Burgt I. Noonan syndrome. Orphanet J Rare Dis 2007;2:4.

3. Tartaglia M, Mehler EL, Goldberg R, et al. Mutations in PTPN11, encoding the protein tyrosine phosphatase SHP-2, cause Noonan syndrome. Nat Genet 2001:29:465-468.

4. Roberts AE, Araki T, Swanson KD, et al. Germline gain-of-function mutations in SOS1 cause Noonan syndrome. Nat Genet 2007;39:70-74.

5. Tartaglia M, Pennacchio LA, Zhao C, et al. Gain-of-function SOS1 mutations cause a distinctive form of Noonan syndrome. Nat Genet 2007;39:75-79.

6. Pandit B, Sarkozy A, Pennacchio LA, et al. Gain-of-function RAF1 mutations cause Noonan and LEOPARD syndromes with hypertrophic cardiomyopathy. Nat Genet 2007;39:1007-1012.

7. Razzaque MA, Nishizawa T, Komoike $Y$, et al. Germline gain-of-function mutations in RAF1 cause Noonan syndrome. Nat Genet 2007;39:1013-1017.

8. Aoki Y, Niihori T, Banjo T, et al. Gain-of-function mutations in RIT1 cause Noonan syndrome, a RAS/MAPK pathway syndrome. Am J Hum Genet 2013;93: 173-180.

9. Schubbert S, Zenker M, Rowe SL, et al. Germline KRAS mutations cause Noonan syndrome. Nat Genet 2006;38:331-336.

10. Cirstea IC, Kutsche K, Dvorsky R, et al. A restricted spectrum of NRAS mutations causes Noonan syndrome. Nat Genet 2010;42:27-29.

11. Flex E, Jaiswal $M$, Pantaleoni $F$, et al. Activating mutations in RRAS underlie a phenotype within the RASopathy spectrum and contribute to leukaemogenesis. Hum Mol Genet 2014;23:4315-4327.

12. Martinelli S, De Luca A, Stellacci E, et al. Heterozygous germline mutations in the CBL tumor-suppressor gene cause a Noonan syndrome-like phenotype. Am J Hum Genet 2010;87:250-257.

13. Yamamoto GL, Aguena M, Gos M, et al. Rare variants in SOS2 and LZTR1 are associated with Noonan syndrome. J Med Genet 2015;52:413-421.

14. Cordeddu V, Di Schiavi E, Pennacchio LA, et al. Mutation of SHOC2 promotes aberrant protein $\mathrm{N}$-myristoylation and causes Noonan-like syndrome with loose anagen hair. Nat Genet 2009;41:1022-1026.

15. Chen PC, Yin J, Yu HW, et al. Next-generation sequencing identifies rare variants associated with Noonan syndrome. Proc Natl Acad Sci USA 2014;111: 11473-11478.

16. Vissers LE, Bonetti M, Paardekooper Overman J, et al. Heterozygous germline mutations in A2ML1 are associated with a disorder clinically related to Noonan syndrome. Eur J Hum Genet 2015;23:317-324.

17. Nava C, Hanna N, Michot C, et al. Cardio-facio-cutaneous and Noonan syndromes due to mutations in the RAS/MAPK signalling pathway: genotype- phenotype relationships and overlap with Costello syndrome. J Med Genet 2007:44:763-771.

18. Nyström AM, Ekvall S, Berglund E, et al. Noonan and cardio-facio-cutaneous syndromes: two clinically and genetically overlapping disorders. J Med Genet 2008:45:500-506.

19. Sarkozy A, Carta C, Moretti S, et al. Germline BRAF mutations in Noonan, LEOPARD, and cardiofaciocutaneous syndromes: molecular diversity and associated phenotypic spectrum. Hum Mutat 2009;30:695-702.

20. Yoon S, Seger R. The extracellular signal-regulated kinase: multiple substrates regulate diverse cellular functions. Growth Factors 2006;24:21-44.

21. Colicelli J. Human RAS superfamily proteins and related GTPases. SCi STKE 2004;2004:RE13.

22. Rauen KA. The RASopathies. Annu Rev Genomics Hum Genet 2013;14: 355-369.

23. Gos M, Fahiminiya S, Poznański J, et al. Contribution of RIT1 mutations to the pathogenesis of Noonan syndrome: four new cases and further evidence of heterogeneity. Am J Med Genet A 2014;164A:2310-2316.

24. Koenighofer M, Hung CY, McCauley JL, et al. Mutations in RIT1 cause Noonan syndrome - additional functional evidence and expanding the clinical phenotype. Clin Genet 2016;89:359-366.

25. Bertola DR, Yamamoto GL, Almeida TF, et al. Further evidence of the importance of RIT1 in Noonan syndrome. Am J Med Genet A 2014;164A:2952-2957.

26. Justino $A$, Dias $P$, João Pina $M$, et al. Comprehensive massive parallel DNA sequencing strategy for the genetic diagnosis of the neuro-cardio-faciocutaneous syndromes. Eur J Hum Genet 2015;23:347-353.

27. Nemcikova M, Vejvalkova S, Fencl F, Sukova M, Krepelova A. A novel heterozygous RIT1 mutation in a patient with Noonan syndrome, leukopenia, and transient myeloproliferation-a review of the literature. Eur J Pediatr 2016;175:587-592.

28. Kromeyer-Hauschild K, Wabitsch $M$, Kunze $D$, et al. Perzentile für den Bodymass-Index für das Kindes- und Jugendalter unter Heranziehung verschiedener deutscher Stichproben. Monatsschr Kinderheilkd 2001;149:807-818.

29. Allanson JE, Bohring A, Dörr HG, et al. The face of Noonan syndrome: Does phenotype predict genotype. Am J Med Genet A 2010;152A:1960-1966.

30. Richards S, Aziz N, Bale S, et al.; ACMG Laboratory Quality Assurance Committee. Standards and guidelines for the interpretation of sequence variants: a joint consensus recommendation of the American College of Medical Genetics and Genomics and the Association for Molecular Pathology. Genet Med 2015;17:405-424.

31. Kircher M, Witten DM, Jain P, O'Roak BJ, Cooper GM, Shendure J. A general framework for estimating the relative pathogenicity of human genetic variants. Nat Genet 2014;46:310-315.

32. Gripp KW, Hopkins E, Sol-Church K, et al. Phenotypic analysis of individuals with Costello syndrome due to HRAS p.G13C. Am J Med Genet A 2011;155A: 706-716.

33. Kobayashi T, Aoki Y, Niihori T, et al. Molecular and clinical analysis of RAF1 in Noonan syndrome and related disorders: dephosphorylation of serine 259 as the essential mechanism for mutant activation. Hum Mutat 2010;31: 284-294.

34. Prendiville TW, Gauvreau K, Tworog-Dube E, et al. Cardiovascular disease in Noonan syndrome. Arch Dis Child 2014;99:629-634.

35. Wilkinson JD, Lowe AM, Salbert BA, et al. Outcomes in children with Noonan syndrome and hypertrophic cardiomyopathy: a study from the Pediatric Cardiomyopathy Registry. Am Heart J 2012;164:442-448.

36. van der Burgt I, Kupsky W, Stassou S, et al. Myopathy caused by HRAS germline mutations: implications for disturbed myogenic differentiation in the presence of constitutive HRas activation. J Med Genet 2007:44:459-462.

37. Berger AH, Imielinski M, Duke F, et al. Oncogenic RIT1 mutations in lung adenocarcinoma. Oncogene 2014;33:4418-4423.

38. Cancer Genome Atlas Research Network. Comprehensive molecular profiling of lung adenocarcinoma. Nature 2014;511:543-550.

39. Gómez-Seguí I, Makishima H, Jerez A, et al. Novel recurrent mutations in the RAS-like GTP-binding gene RIT1 in myeloid malignancies. Leukemia 2013;27:1943-1946

40. Kratz CP, Franke $\mathrm{L}$, Peters $\mathrm{H}$, et al. Cancer spectrum and frequency among children with Noonan, Costello, and cardio-facio-cutaneous syndromes. Br J Cancer 2015:112:1392-1397. 